\title{
A novel concept of convertible roofs with high transformability consisting of planar scissor-hinge structures
}

\author{
Yenal Akgün ${ }^{\mathrm{a}, *}$, Charis J. Gantes ${ }^{\mathrm{b}}$, Konstantinos E. Kalochairetis ${ }^{\mathrm{b}}$, Gökhan Kiper ${ }^{\mathrm{c}}$ \\ a Department of Architecture, Izmir Institute of Technology, Gülbahçe Kampüsü 35430 Izmir, Turkey

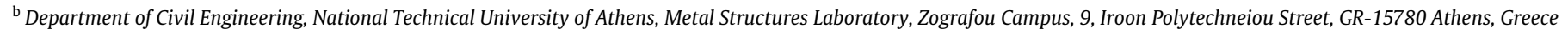 \\ ${ }^{\mathrm{c}}$ Department of Mechanical Engineering, Middle East Technical University, 06531 Ankara, Turkey
}

\section{A R T I C L E I N F O}

\section{Article history:}

Received 30 November 2009

Received in revised form 4 May 2010

Accepted 10 May 2010

Available online 9 June 2010

\section{Keywords:}

Scissor-hinge structures

Deployable structures

Convertible roofs

Kinetic structures

Transformability

Geometrically nonlinear finite element analysis

\begin{abstract}
A B S T R A C T
In this paper, a new adaptive scissor-hinge structure is introduced, which can be converted by means of actuators between a multitude of curvilinear arch-like shapes, where it can be stabilized and carry loads. The key point of this new structure is the proposed Modified Scissor-Like Element (M-SLE). With the development of this element, it becomes possible to change the geometry of the whole system without changing the dimensions of the struts or the span. The proposed scissor-hinge structure discussed here is planar, but it is also possible to combine structures in groups to create spatial systems. After outlining the differences of the proposed structure with existing designs, the dimensional properties of the M-SLE are introduced. Then, geometric principles and shape limitations of the whole structure are explained. Finally, structural analysis of the structure in different geometric configurations is performed, in order to discuss stiffness limitations associated with the advantage of increased mobility.
\end{abstract}

(c) 2010 Elsevier Ltd. All rights reserved.

\section{Introduction}

Deployable structures can be defined as structures that can be transformed from a closed compact configuration to a predetermined, expanded form, in which they are stable and can carry loads [1]. According to this definition, a deployable structure should have predefined deployed and retracted geometric shapes. When current examples of deployable structures are studied, this definition can indeed be confirmed. Thus, all such structures have definite "open-closed" body forms; and transformations occur between these two body forms by using one of the various transformation types, such as sliding, deploying, rotating, folding and their variations [2]. Fig. 1 shows various transformation types of existing deployable structures in a schematic way.

Scissor-hinge structures are one of the most common types of deployable structures. Most of the research associated with scissor-hinge structures deals with two main topics: Obtaining common forms via different folding types; and/or obtaining defined forms by using structural elements with different geometries or materials.

Pinero's research in 1961 [3] is one of the earliest studies on this topic. Pinero invented a scissor mechanism, in which each rod

\footnotetext{
* Corresponding author. Tel.: +90 5066658168.

E-mail address: yenalakgun@gmail.com (Y. Akgün).
}

has three pivot joints, one on each end and one in between. As two ends of a scissor mechanism are brought together, the centre pivots are spread apart, thus lengthening the mechanism as a whole to a planar pattern. In addition, Pinero realized that if the interior pivot point on a rod is not at the midpoint, then it is possible to create a shell-shaped surface. Following Pinero, Escrig and Valcarcel focused on the development of new spatial grids and patterns for common arches, domes and large scale umbrellas [4-7]. During this development, they generally concentrated on new connection (hinge) elements. Their studies proved that a defined geometry can be obtained from the multiplication of different units. In addition to the development of geometric designs for new structures, Escrig's [8,9], Gantes' [10-14] and Langbecker's works $[15,16]$ also aimed at explaining the structural behaviour of the scissor-hinge structures in analytical and numerical ways, and at proposing methods for their combined geometric and structural design. However, the work of these researchers also concentrated on structures that are transformed between two pre-defined geometries, an open and a closed one, without attempting to provide additional geometric flexibility of scissor-hinge structures.

Hoberman [17] made a considerable advance in the design of scissor-hinge structures, when he discovered the simple angulated element. By using this element, Hoberman created the transformable Iris Dome. In the Iris Dome angulated elements form a circular shape in plan and the joints connecting their end nodes 

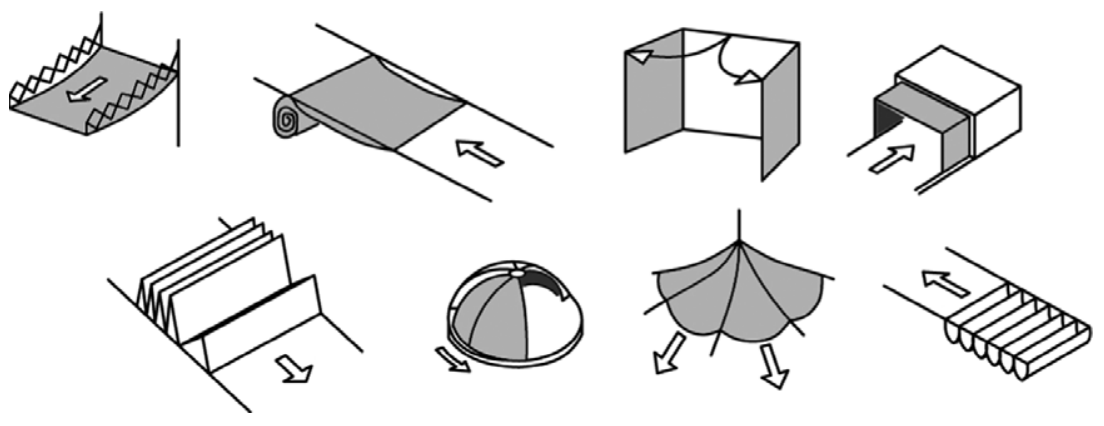

Fig. 1. Transformation types of various kinetic systems.

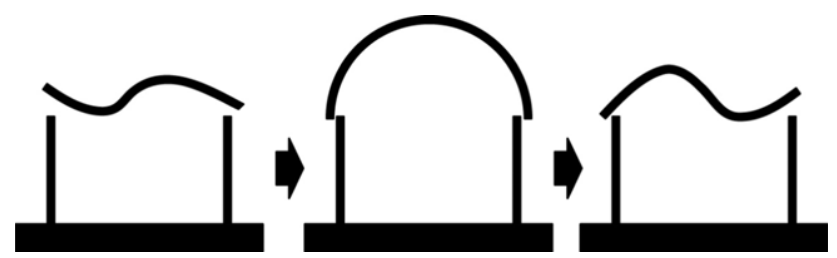

Fig. 2. Schematic sections of the proposed convertible roof.

connect the circles to each other. This allows the structure to transform toward its perimeter, thus creating a central opening at the centre when transformed. Pellegrino and his group (DSL), among other extensive research work on deployable structures, also developed the geometric principles of this angulated element [18], and applied the principles of scissor-hinge structures to plates [19].

Calatrava proposed new structural units and mechanisms in his Ph.D. thesis [20], and achieved increased geometric flexibility with his convertible projects and sculptures [21]. Kokawa's Cable Scissors Arch (CSA) [22] uses scissors as a convertible structure that can change its geometry. CSA consists of three-hinged arch scissors and zigzag flexible cables with pulleys installed at the connection points between the scissor units. By winding up the cable by a winch, CSA expands and is forced to lift up. It can be shortened and go down by its self-weight during the winding back. However, this structure can only meet symmetric geometries, which is not sufficient for a fully flexible structure.

All the above mentioned examples can basically convert their shapes during the opening or closing processes. However, even though some parts of these structures do move, rotate or slide, it can be claimed that those examples are insufficient for constituting real form flexibility. There is a need for adaptive structures that can convert between more than two different geometries, thus offering more flexible form alternatives [23]. The proposed convertible structure meets this need, as it can be transformed between continuous regimes of arch-like curvilinear geometries. Fig. 2 shows some schematic sections of the proposed convertible roof.

To arrive at this convertible roof structure, the present study considers the use of scissor-hinge structures combined with actuators. Scissor-hinge structures possess unique extension and rotation capabilities, and the Modified Scissor-Like Element (M-SLE) developed herein greatly increases the form possibilities of the structure. With the development of this modified element, it is possible to change the geometry of the whole system without changing the dimensions of the struts or the span.

The proposed scissor structure can be useful for a wide range of applications. For example, such a kinetic structure can be used as roof of an exhibition hall. Depending on the activities in that hall, the shape of the roof can be transformed by the users. This kind of a transformation offers great flexibility for spaces. Another example of application of such structures is that of solar roofs that can rotate according to the location of the sun, thus having increased productivity in comparison to conventional solar panels. Scissor

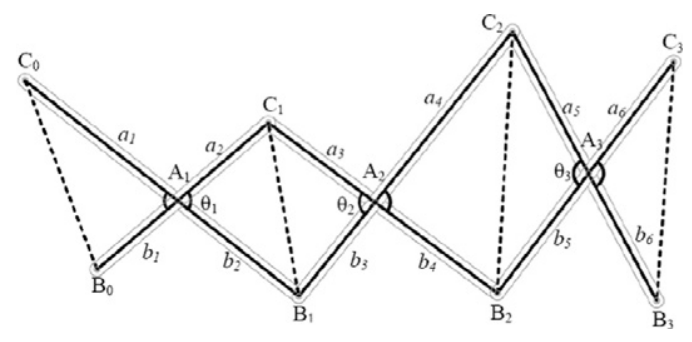

Fig. 3. Deployability condition for a sample scissor structure.

structures can be conveniently connected to control systems, so by using appropriate motors the required transformations can be performed easily.

To understand the principles of the proposed structure, first, general characteristics of scissor-hinge structures are summarized. Then, the proposed structure is introduced, the developed modified scissor element is presented, and the associated transformation capabilities are explained. The geometric constraint equations are derived and a geometric design methodology is proposed. Finally, a series of structural analyses are carried out at different geometric configurations, and the results are studied, in order to understand to what extend increased geometric flexibility is achieved at the expense of reduced stiffness and strength.

\section{Common scissor-hinge structures: definitions and typolo- gies}

To form a simple scissor element, two bars are connected to each other at an intermediate point through a pivotal connection, allowing them to rotate freely about an axis perpendicular to their common plane but restraining all other degrees of freedom. This element is called scissor-like element (SLE) [1]. All scissor-hinge structures arise from the concatenation of SLEs. SLEs can be put together into almost any configuration, but in this paper, only planar structures with straight bars are considered.

One of the most important requirements of the scissor-hinge structures is that the configuration is able to be contracted into a compact shape. Fig. 3 shows a basic scissor-hinge structure. It can be deduced that in the compact shape, the three elements will have theoretically one dimension, and $C_{0}, B_{0}, A_{1}, C_{1}, B_{1}, A_{2}, C_{2}, B_{2}$, $A_{3}, C_{3}, B_{3}$ will be co-linear. To meet this condition, the sum of the lengths of the bar segments on either side of any SLE, should be equal to the sum of the lengths of the corresponding bar segments of the adjacent SLEs $[1,15]$ :

$a_{i-1}+b_{i-1}=a_{i}+b_{i}$.

Scissor-hinge structures can be classified into two categories, based on the properties of the lines connecting their intersection points. The first group consists of translational scissor-hinge structures, which can only slide without any rotation. This condition is met if all axes connecting the pairs of hinges between adjacent SLEs 
a

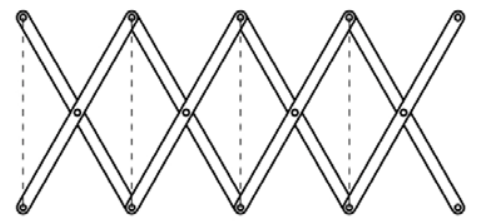

b

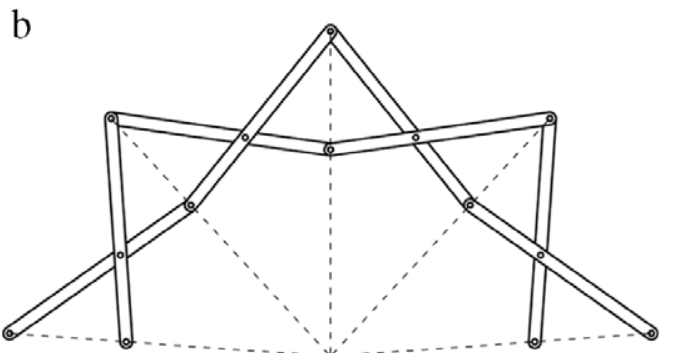

Fig. 4. Translational (a) and curvilinear (b) scissor-hinge systems.

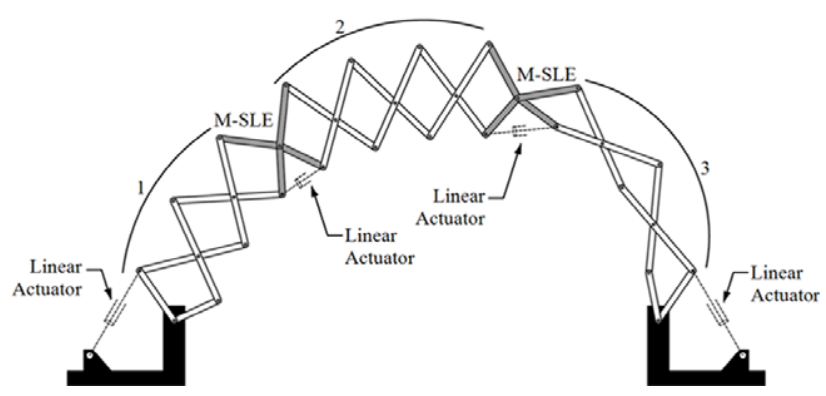

Fig. 5. Locations of M-SLEs and actuators on proposed scissor-hinge structure at a random geometric configuration.

are parallel to each other (Fig. 4(a)). The second group consists of curvilinear scissor-hinge structures, where the above axes intersect at one or more common points and are rotated around their intersection points during deployment (Fig. 4(b)). Both of these groups can only transform along one axis; so it is only possible to obtain contracted, deployed and some semi-deployed, intermediate forms. This, however, is considered as offering partial only and not full flexibility.

\section{Topological and geometric properties of proposed scissor- hinge structure}

The proposed scissor-hinge structure is curvilinear. Moreover, for facilitating the calculations and optimization of the structural lengths, all SLEs have the same dimensions, and $a_{i} / b_{i}$ has a constant value. The desired geometric flexibility is achieved by introducing the so-called Modified Scissor-Like Elements (M-SLEs), which will be presented next. In Fig. 5, the main elements of the proposed scissor-hinge structure, including M-SLEs, can be seen. In these structures, the number of M-SLEs depends on the desired transformability and is chosen accordingly; the number and dimensions of simple SLEs depends on the span. In order to understand the structure better, M-SLEs are explained in the following section.

\subsection{Modified Scissor-Like Element (M-SLE)}

The key point of the proposed scissor-hinge structure is the Modified Scissor-Like Element (M-SLE), illustrated in Fig. 6. An M-SLE is obtained by the connection of four struts by three hinges on a common point. Thus, each of the four struts can rotate freely

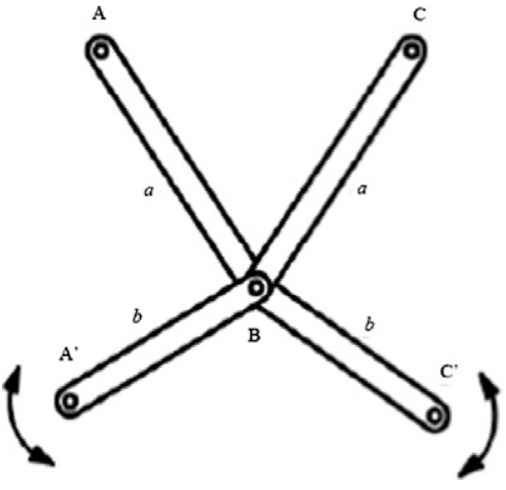

Fig. 6. Modified Scissor-Like Element (M-SLE).

about their common point, without affecting the other three struts. While the number of degrees of freedom (DoF) of an SLE is equal to one, the corresponding number of DoFs of an M-SLE is equal to three. Hence, when an M-SLE is used in a scissor-hinge structure, it increases the number of DoFs of the whole system, thus enabling the system to change its shape, without changing the span length or the dimensions of the bars.

In Fig. 5, possible locations of M-SLEs on a planar scissor-hinge structure can be seen. Two M-SLEs are used, increasing the mobility of the system by $2 \times 2=4$, thus four actuators are needed for having full geometric control of the system and for stabilizing it at any desired geometric configuration.

All scissor-hinge structures between two M-SLEs or between one M-SLE and a support point behave consistently. This means that, when one of these SLEs moves, all other SLEs follow this movement. However, in the proposed structure, M-SLEs divide the whole system into sub-structures, acting as "isolators" between them, so that each sub-structure can transform without directly affecting the other sub-structures. For example, in the system of Fig. 5, there are two M-SLEs, dividing the whole structure into three "isolated" parts. Thus, movement of one SLE in one group is followed only by the SLEs in this group, not by the others. This independency of the sub-groups creates the desired additional geometric mobility.

\subsection{Transformation capability of the proposed structure}

The transformation capability of the whole structure is related to three factors: First, the number of M-SLEs in the system; second, the dimensions of the SLEs and M-SLEs; third, the support points. These three factors are analyzed next.

\subsubsection{Transformation capability according to the number of M-SLES}

For the proposed structure, the number of M-SLEs is the most important factor that affects its transformation capacity. All SLEs and M-SLEs in Fig. 7(a) and (b) have the same dimensional properties. The only difference is that the structure in Fig. 7(a) has one M-SLE, while the structure in Fig. 7(b) has two. Due to this difference, the number of independent sub-structures changes from two to three; and this affects the transformation capacity of the system. The shape difference between the two examples can be observed by investigating the curves passing through the pivot points. The increased number of M-SLEs enhances the transformation capacity, but also leads to an increase of the required number of actuators and the overall cost of the system. For this reason, the minimum number of M-SLEs must be used that are needed for achieving the desired forms. In the examples presented hereafter, two M-SLEs are used, as a balance between transformation capacity and complexity/cost of the system. 


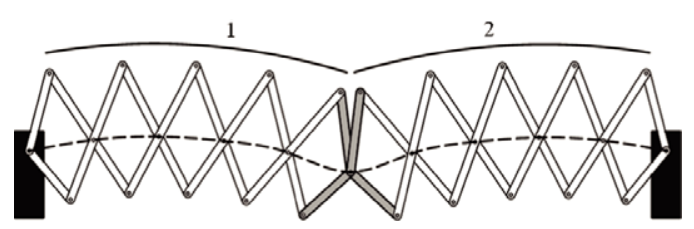

(a) The system with one M-SLE.

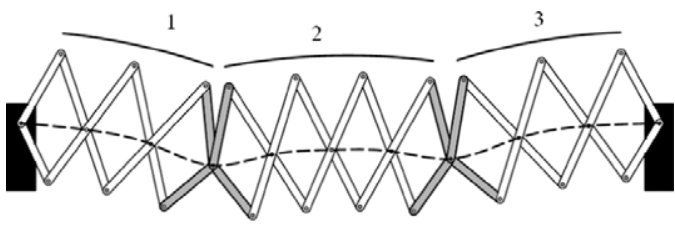

(b) The system with two M-SLEs.

Fig. 7. Geometric options according to the number of M-SLEs.

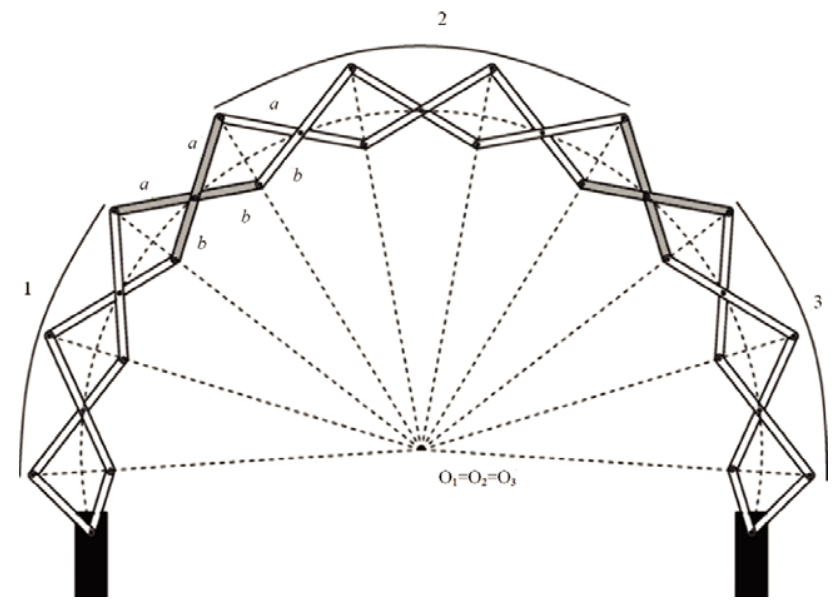

(a) Circular arch.

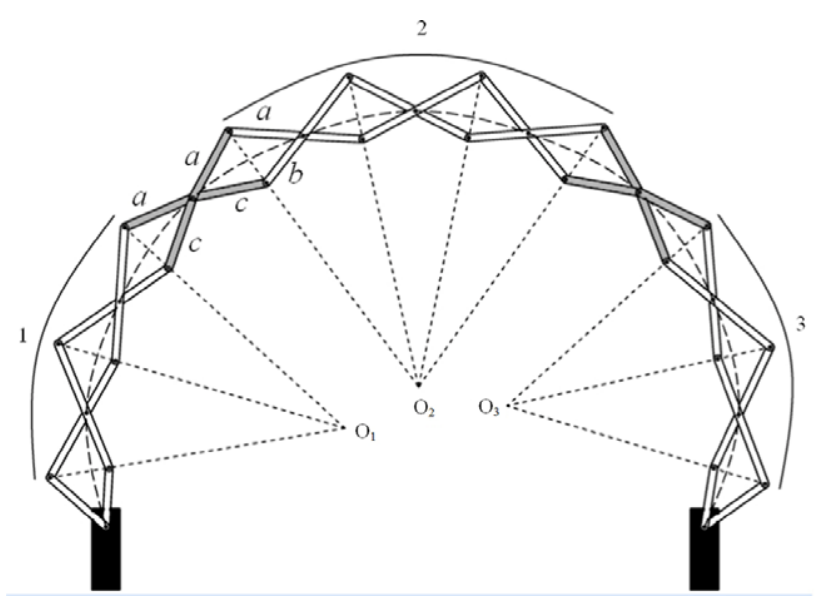

(b) Non-circular arch.

Fig. 8. Geometric options according to the dimensions of M-SLEs.

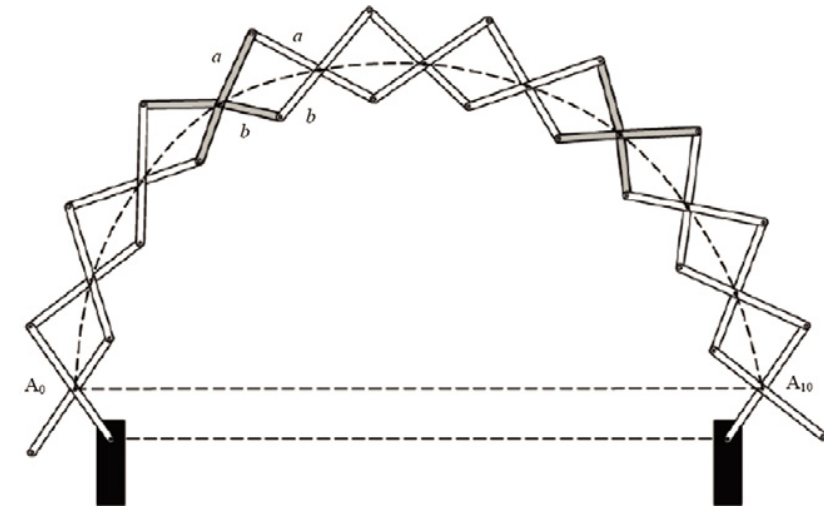

(a) System supported on the ground at hinges.

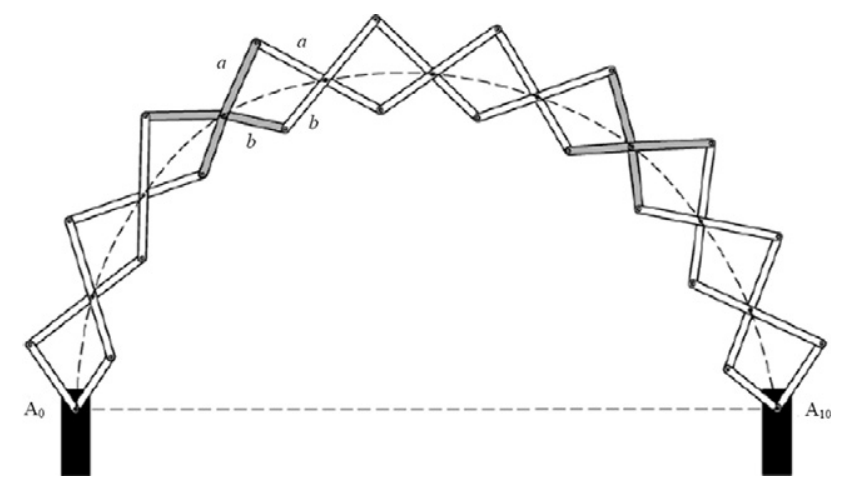

(b) System supported on the ground at pivots.

Fig. 9. Alternative support conditions.

3.2.2. Transformation capability according to the dimensions of $M$ SLES

There are two options for the dimensions of M-SLEs. In the first one, M-SLEs have the same dimensions as the other SLEs in the system and all axes connecting hinge points intersect at one point (Fig. 8(a)). Thus, the structure constitutes part of a circular arch. In the second option, M-SLEs are dimensionally different from other SLEs $(c \neq b)$. Then, the axes of the three independent substructures intersect at three different points, and the overall structure can never constitute part of a circular arch (Fig. 8(b)).

\subsubsection{Transformation capability according to the support points}

The transformation capability of the proposed structure as well as its overall behaviour can also change according to the support points. In Fig. 9 two alternatives are offered. The system in Fig. 9(a) is supported on the ground at a hinge. Due to this large shear forces occur in the corresponding bottom bars on each side. The system in Fig. 9(b) is supported on the ground at a pivot, thus avoiding the previous disadvantage. This is why structures that connect to the ground at pivot points were used in this study.

\subsection{Kinematic analysis of the proposed structure}

\subsubsection{Kinematic analysis of a single scissor-like element}

The shape limitations of one SLE directly affect the kinematics of the whole system, so that geometric analysis of a single element should be performed first. In Fig. 10, two successive identical SLEs can be seen. From experimental studies on physical models, it was observed that when $\left(A_{1} B_{1} A_{2}\right)$ angle $\left(\theta_{1}^{\prime}\right)$ becomes smaller than $180^{\circ}$, the whole system becomes unstable. Thus, this angle must be larger than $180^{\circ}$ for all SLE pairs. It is then possible to calculate the upper and lower bounds of $\left(\gamma_{1}\right)$ and $\left(\alpha_{1}\right)$ angles. 


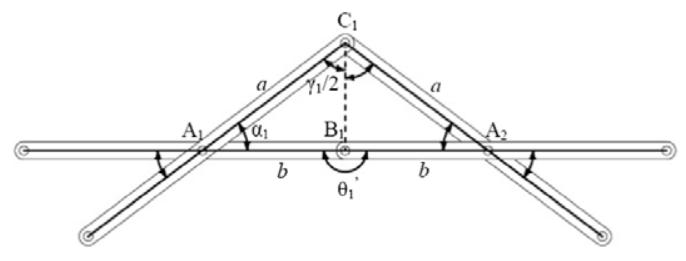

Fig. 10. Definition of angles for a single Scissor-Like Element.

According to Fig. 10, it can be obtained that;

$$
\begin{aligned}
& 180^{\circ} \geq \theta_{1} \geq 0^{\circ} \\
& 180^{\circ} \geq \alpha_{1} \geq \cos ^{-1}\left(\frac{b}{a}\right) \\
& 0^{\circ} \leq \frac{\gamma_{1}}{2} \leq \sin ^{-1}\left(\frac{b}{a}\right) .
\end{aligned}
$$

\subsubsection{Kinematic analysis of the whole system}

As mentioned above, the proposed structure requires actuators that control its shape during transformation and are then used for fixing it at the desired positions. The mobility of a mechanical system describes the number of additional actuators needed to fix or move the system safely. For all kinetic structures, mobility of the system is larger than or equal to one $(M \geq 1)$. If the mobility of a kinematic system is equal to zero $(M=0)$, the structure is stable and requires no additional stabilization [24]. There are different formulas to calculate the mobility of different structural systems, but in this work Freudenstein-Alizade approach [25] for structural groups has been adopted, according to which:

$M=J_{T}-\lambda L+q-j_{p}$

where the term $J_{T}$ includes the contribution of DoFs of all joints, $\lambda$ denotes the active DoFs of the space where the mechanism operates ( $\lambda=3$ for planar systems and $\lambda=6$ for spatial systems), $L$ is the number of loops at the system, $q$ is the number of overconstrained links, and $j_{p}$ describes the passive mobilities in the joints.

For applying this formula to the structure in Fig. 5 (repeated with additional notation in Fig. 11), it can be seen that the structure has $J_{T}=37$ joints, and $L=11$ independent loops (where the 11 th loop is the one closing the system). Moreover, there are no overconstrained links $(q=0)$, and $j_{p}$ no passive mobilities in the joints $\left(j_{p}=0\right)$. Thus:

$M=37-3 \times 11=4$.

The kinematic analysis of the mechanism shall be carried out in two parts: direct kinematics and inverse kinematics. Direct kinematics refers to determining the configuration of the system given the input parameters, while inverse kinematics refers to calculating the necessary input parameter values for a desired predetermined configuration. For practical use, the inverse kinematics problem has to be solved, while the direct kinematics problem is necessary for workspace analysis. In the following, first the details of the direct kinematics algorithm are presented and then an algorithm for the inverse kinematics problem is proposed.

The proposed structure consists of three sub-structures. When the input parameters are selected, such that three of them drive the substructures independently, an analytical solution for direct kinematics is possible. However, when the actuators couple two substructures, as is the case in the structure of Fig. 5, an analytical solution is not possible and it is required to solve a highly nonlinear system of equations with four unknowns. Hence, it is not desirable to use these actuators in the direct kinematics of the problem.

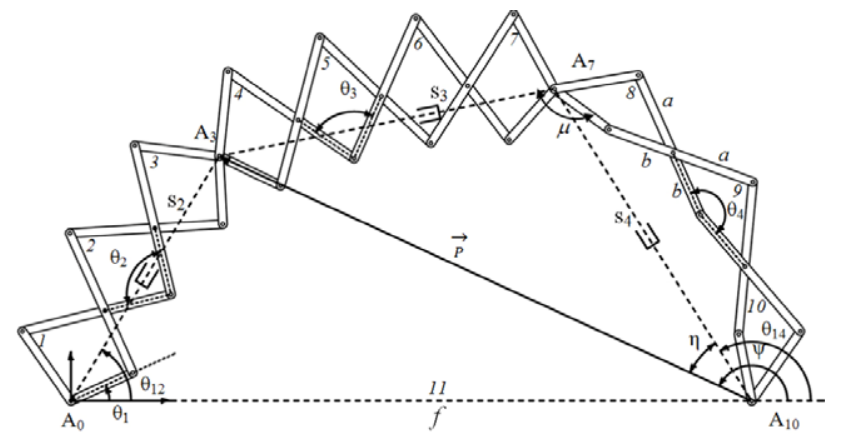

Fig. 11. Kinematic analysis variables for the proposed planar scissor-hinge structure.

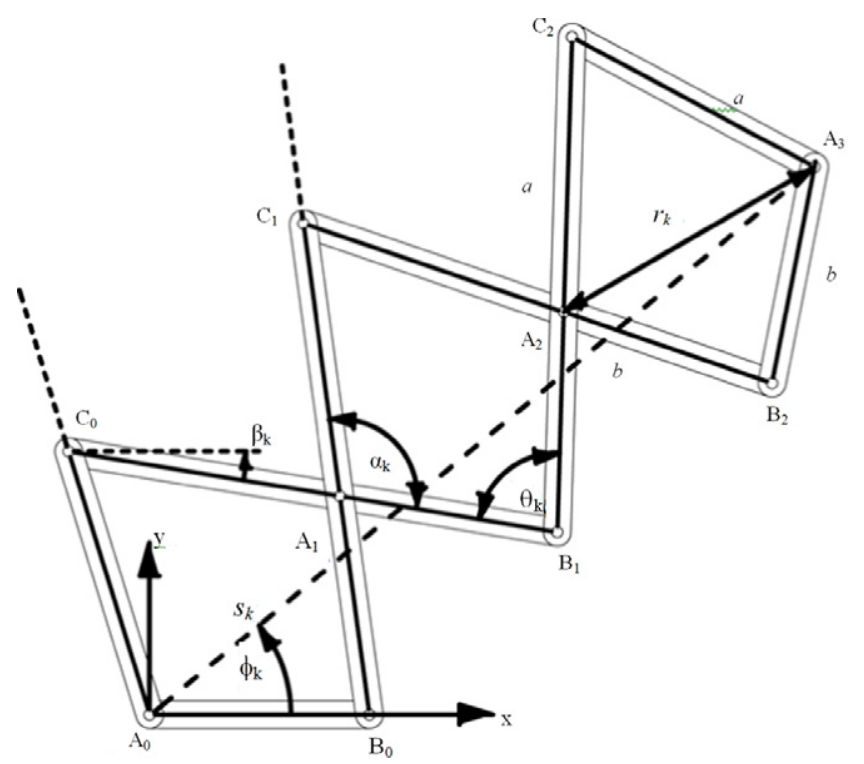

Fig. 12. Variable parameters in one scissor module.

However, as shall be clear in the relevant sections, if an appropriate set of input parameters is used for the direct kinematics, the inverse kinematics for any set of inputs as well as the workspace analysis can be performed.

The structure in Fig. 11 can be abstracted to a mechanism with 7 links, namely 4 revolute $(R)$ joints, allowing rotation, and 3 prismatic $(\mathrm{P})$ joints, allowing pantograph motion, which is called an RPRPRPR mechanism. In Fig. $11, A_{0} A_{10}$ (of length $f$ ) is the fixed link and the three link pairs between $A_{0}-A_{3}, A_{3}-A_{7}$ and $A_{7}-A_{10}$ are joined to each other by prismatic joints. Due to these prismatic joints, the distances $\left|A_{0} A_{3}\right|,\left|A_{3} A_{7}\right|$ and $\left|A_{7} A_{10}\right|$ are represented by the joint variables $s_{2}, s_{3}$ and $s_{4}$, respectively. When $s_{2}, s_{3}$ and $s_{4}$ are given, there is still one free parameter; as such the inclination of $A_{0} A_{3}$ with respect to the base, $\theta_{12}$ shall be selected (Fig. 11). Thus, $\left(\theta_{12}, s_{2}, s_{3}, s_{4}\right)$ is a set of input parameters. Note that these parameters are not actual actuator variables, but are intermediate variables which will be used for the solution of the forward kinematics problem. Yet, for an $M=1$ module with $2 n$ bars (like $A_{0} A_{3}, A_{3} A_{7}$ or $A_{7} A_{10}$ ), determination of the positions $A_{k}$ and the distances $s_{k}(k=2,3, \ldots)$ is possible by using the angles between two connected bars $\left(\alpha_{k}\right.$ or $\left.\theta_{k}\right)$ as the input parameters (Fig. 12). For the structure in Fig. 11, $\theta_{1}, \theta_{2}, \theta_{3}, \theta_{4}$ are selected as input parameters for the whole mechanism; while $\alpha_{k}, \beta_{k}$ and $r_{k}$ variables, defined in Fig. 12, are the variables to be determined. Note that all deltoids $\left(A_{i} B_{i} A_{i+1} C_{i}\right)$ in a module are identical; hence all $\angle A_{i} B_{i} A_{i+1}=\theta_{k}, \angle B_{i} A_{i} C_{i}=\alpha_{k}, \angle B_{i} A_{i} C_{i}=\beta_{k}$ and $\left|A_{i} A_{i+1}\right|=r_{k}$ values are the same in a module $k$. When the law of cosines is 


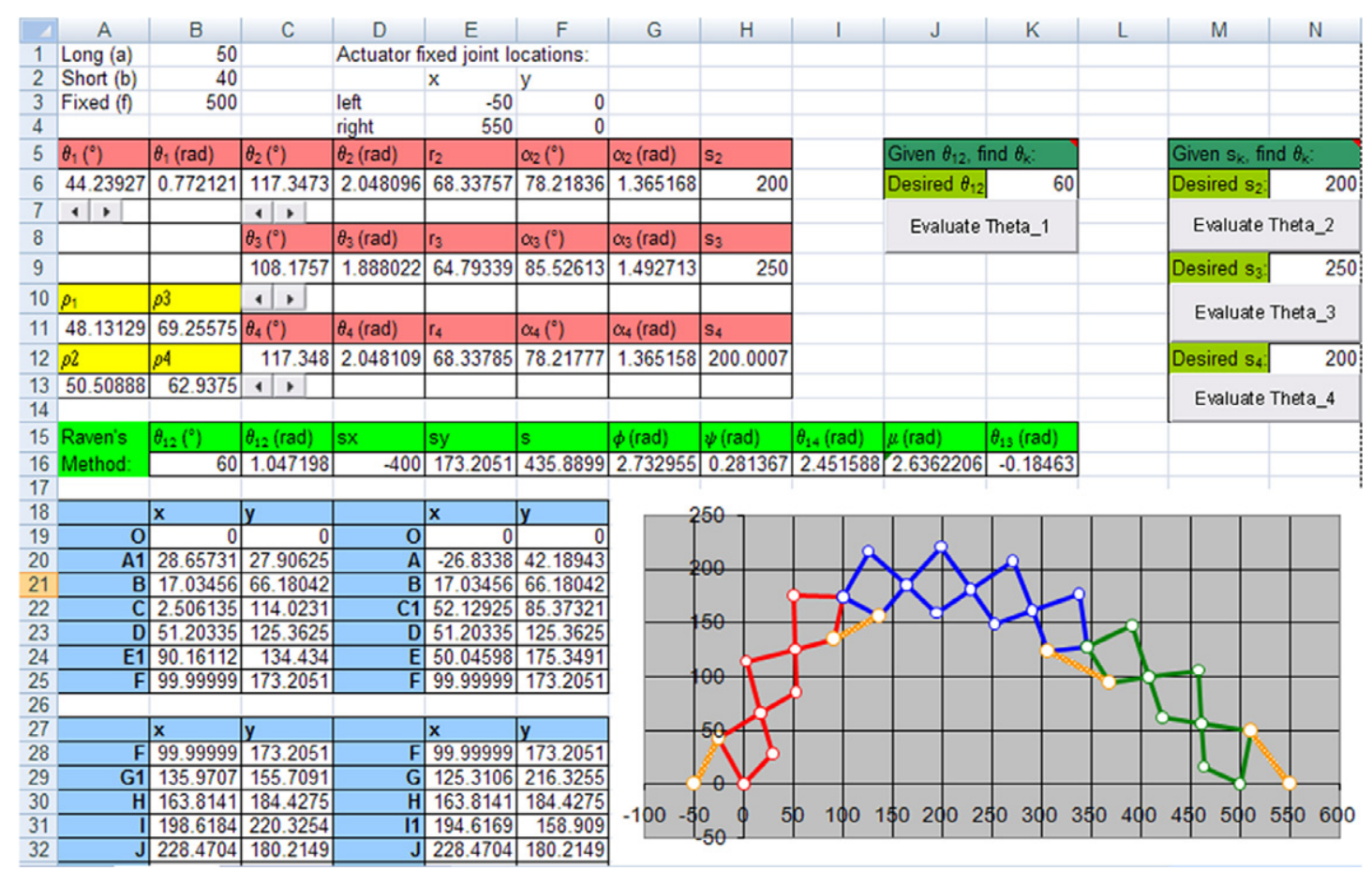

Fig. 13. Interface of the geometric analysis computer program.

applied to triangle $A_{2} B_{2} A_{3}$ in Fig. 12;

$r_{k}=b \sqrt{2-2 \cos \theta_{k}}$.

From the symmetry of the deltoid (from triangles $A_{2} B_{2} A_{3}$ and $\left.A_{2} C_{2} A_{3}\right)$

$\alpha_{k}=\cos ^{-1}\left(\frac{r_{k}}{2 b}\right)+\cos ^{-1}\left(\frac{r_{k}}{2 a}\right)$

$\beta_{k}=\pi-\alpha_{k}-\left(2 \pi-2 \alpha_{k}-\theta_{k}\right)=\alpha_{k}+\theta_{k}-\pi$.

When a scissor module with $n$ deltoids is aligned to the $x$ and $y$ coordinate system, as shown in Fig. $12, A_{0}$ is located at the origin of the coordinate system; and the $k$ th deltoid can be defined in vector notation, using Euler equation, as follows:

$\overrightarrow{A_{0} A_{m+1}}=\overrightarrow{A_{0} A_{m}}+r_{k} \mathrm{e}^{\mathrm{i}\left(\frac{\pi}{2}-\frac{\theta_{k}}{2}-m \beta_{k}\right)}$

$\overrightarrow{A_{0} B_{m}}=\overrightarrow{A_{0} A_{m}}+b \mathrm{e}^{-\mathrm{i} m \beta_{k}}$

$\overrightarrow{A_{0} C_{m}}=\overrightarrow{A_{0} A_{m}}+a \mathrm{e}^{\mathrm{i}\left(\alpha_{k}-m \beta_{k}\right)} \quad(m=0, \ldots, n-1)$.

When Eqs. (10)-(12) are investigated, it is seen that every deltoid rotates by $\beta_{k}$ with respect to the previous one. From this information;

$s_{k}=\overrightarrow{\mid A_{0} A_{n}} \mid, \quad \varphi_{k}=\left\langle\overrightarrow{A_{0} A_{n}}\right.$

When lengths $s_{2}, s_{3}, s_{4}$ are calculated, by knowing the fixed length $f$ between supports, the system can be thought as a typical four-bar mechanism $A_{0} A_{3} A_{7} A_{10}$. This mechanism can be analyzed by using Raven's method [26] (see Fig. 11):

$$
\begin{aligned}
& \theta_{12}=\theta_{1}+\phi_{1}, \quad P_{x}=f-s_{2} \cos \left(\theta_{12}\right), \quad P_{y}=s_{2} \sin \left(\theta_{12}\right) \\
& P=\sqrt{P_{x}^{2}+P_{y}^{2}, \quad \psi=L \vec{P}} \\
& \eta=\cos ^{-1}\left(\frac{p^{2}+s_{4}^{2}-s_{3}^{2}}{2 P s_{4}}\right), \quad \theta_{14}=\psi-\eta \\
& \mu=\cos ^{-1}\left(\frac{s_{3}^{2}+s_{4}^{2}-p^{2}}{2 s_{3} s_{4}}\right), \quad \theta_{13}=\theta_{14}-\mu .
\end{aligned}
$$

This completes the direct kinematics, given $\theta_{1}, \theta_{2}, \theta_{3}$ and $\theta_{4}$. The above kinematic analysis has been implemented in Microsoft Excel $2003^{\circledR}$. The link lengths $a, b$ and $f$ can be varied in this analysis platform and the structure can be simulated by changing input parameter values using spin buttons. In Fig. 13, the interface of this program is depicted.

The inverse kinematics problem is handled with the following algorithm:

- Construct the RPRPRPR mechanism according to the desired shape; hence obtain $\theta_{12}, s_{2}, s_{3}$ and $s_{4}$.

- Find $\theta_{k}$ for given $\theta_{12}$ and $s_{k}$ using a numerical algorithm, such as the Newton Raphson method (in Microsoft Excel 2003 "goal seek" tool can be used).

- Perform the kinematic analysis with $\theta_{1}, \theta_{2}, \theta_{3}, \theta_{4}$.

- Obtain the required values for any parameter, such as the linear actuator lengths in Fig. 5.

With this algorithm, any desired input parameter value can be found for a given configuration. Characteristic geometric configurations of a structure of this type, as obtained from the software described above, are illustrated in Fig. 14.

The following example is presented for clarification of the proposed algorithm: Let the desired shape be such that $\theta_{12}=60^{\circ}$, $s_{2}=200, s_{3}=250$ and $s_{4}=200$. These values are typed to cells B16, N6, N9 and N12 in Fig. 13. Then $\theta_{1}, \theta_{2}, \theta_{3}$ and $\theta_{4}$ are determined one by one using $\theta_{12}, s_{2}, s_{3}$ and $s_{4}$ values by a Newton-Raphson algorithm. These algorithms are utilized via the "Goal-Seek" tool of Excel ${ }^{\circledR}$, embedded in the four command buttons in Fig. 13. Once $\theta_{1}, \theta_{2}, \theta_{3}$ and $\theta_{4}$ are obtained, all joint locations are determined according to Eqs. (7)-(17). Finally, the input lengths $\rho_{1}, \rho_{2}, \rho_{3}$ and $\rho_{4}$ of the linear actuators in Fig. 5 are evaluated.

\section{Static analysis}

As expected, there is a price to be paid for the increased transformation capability of the proposed structure, and this is related besides the cost associated with complicated connections between members, to the reduced stiffness and load bearing capacity in the 

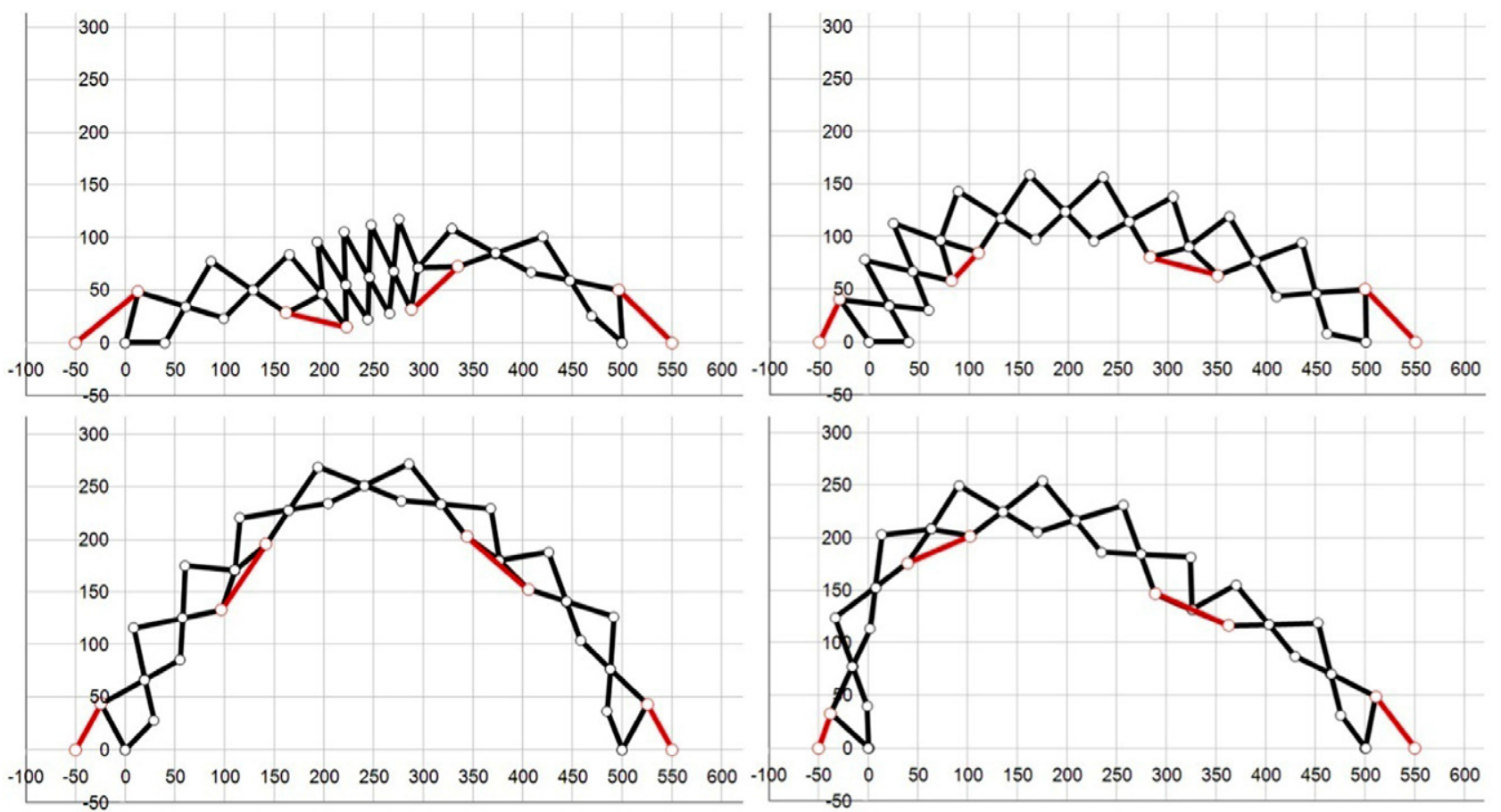

Fig. 14. Successive geometric configurations of the proposed structure.

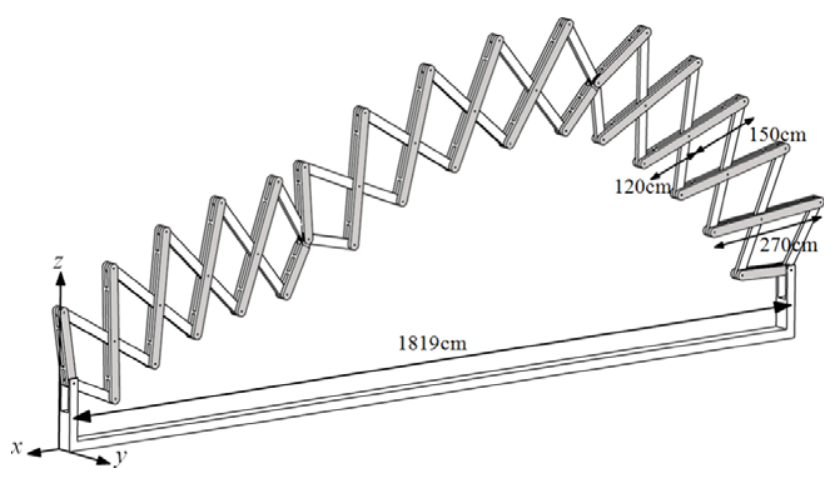

Fig. 15. One configuration of the structure that has been analyzed.

stable, deployed configuration. In order to quantify this disadvantage, a set of structural analyses have been carried out, subjecting the structure to typical loading patterns in different geometric configurations. Additional objectives of the static analysis were to investigate how the locations of actuators influence the stiffness and strength and to find the optimum locations, as well as to obtain the minimum cross-sectional dimensions of the struts.

The structure which has been used for the static analysis (Fig. 15) is formed by 14 identical SLEs and two M-SLEs and has a total span is $1819 \mathrm{~cm}$. Each strut is $270 \mathrm{~cm}$ long and the pivot point of each SLE is located $120 \mathrm{~cm}$ from the bottom node of the strut. Thus, the whole structure is a curvilinear scissor-hinge system of the type described in Fig. 4(b).

In the analysis the response of the structure against in-plane vertical and horizontal loads has been simulated. Three different geometries (high arch, wavelength arch, shallow arch) were analyzed, with four different actuator combinations (See Fig. 16). These three geometries are characteristic of an infinite number of different geometries that the structure can achieve.

Due to the relatively high flexibility, geometric nonlinearity has been taken into account in the analyses, while the material was assumed to be linear elastic, confirming this assumption later on by carrying out elastic checks for cross-sectional and member strength, in accordance with similar studies of flexible structures in the past (for example [27]). S275 steel with an elastic modulus equal to $21000 \mathrm{kN} / \mathrm{cm}^{2}$, Poisson's ratio equal to 0.3 and yield stress equal to $27.5 \mathrm{kN} / \mathrm{cm}^{2}$ was considered. The analyses were performed with the well-known finite element software ADINA. The model consisted of Hermitian beam elements with three degrees of freedom at each end and was suitably discretized in order to obtain sufficient accuracy.

Two typical load cases were considered, one consisting of a predominantly vertical load, which represents self weight and snow load, and the second of a predominantly horizontal load, which represents wind. All loads were applied as concentrated on the exterior nodes. Rectangular hollow cross-sections of $5 \mathrm{~cm} \times$ $30 \mathrm{~cm} \times 1 \mathrm{~cm}$ were employed for all members. Dotted lines in Fig. 16 represent pairs of bars, as seen in Fig. 15, which were modelled by single members with hypothetical cross-sections of $10 \mathrm{~cm} \times 30 \mathrm{~cm} \times 1 \mathrm{~cm}$. Elastic strength checks of normal stresses due to axial force and bending moment were carried out, while a deflection limit of $\operatorname{span} / 200(L / 200=9.1 \mathrm{~cm})$ was used for serviceability checks.

The high arch, expected to be the most efficient structural shape, was analyzed first. In the first alternative solution (Fig. 16, locations 1 ), the structure was connected to the ground via one hinge on each side, two actuators were placed on the exterior of the two bottom SLEs, while the relative rotation of the three substructures was partially restricted by means of two more actuators. This solution proved by the analysis to be efficient for the case of the high arch resulting to acceptable vertical deflection and amount of stress in the cross-sections (Fig. 17).

In the second alternative solution (Fig. 16, locations 2), the structure was connected to the ground via two actuators, while the relative rotation of the three sub-structures was partially restricted by means of two more actuators. The undeformed and deformed shape, as well as the axial force and bending moment diagrams of the structure under vertical loading are shown in Fig. 18. 

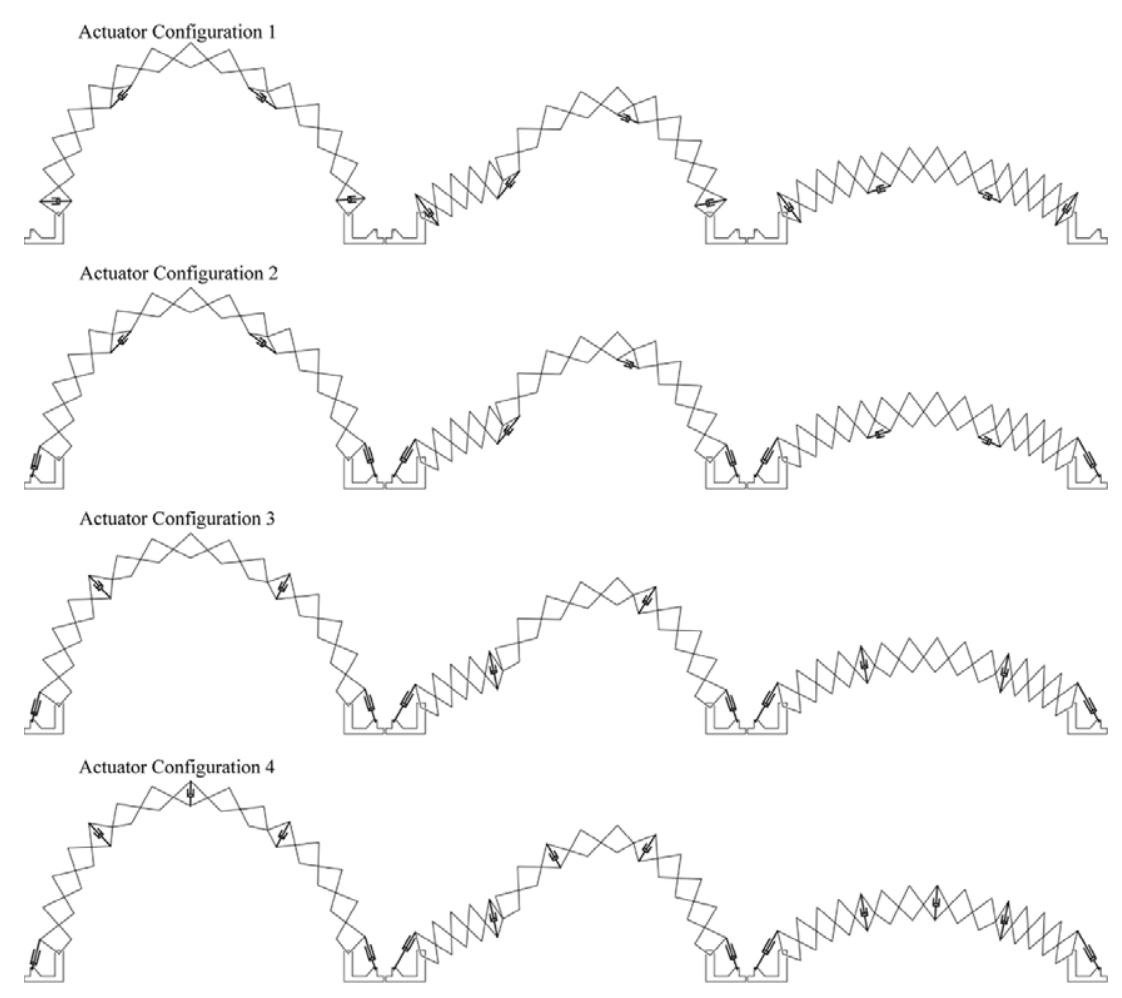

Fig. 16. Tested geometries and alternative locations of actuators.

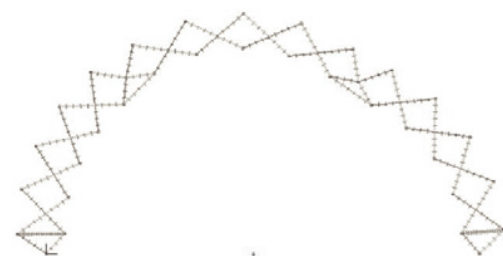

(a) Undeformed shape.

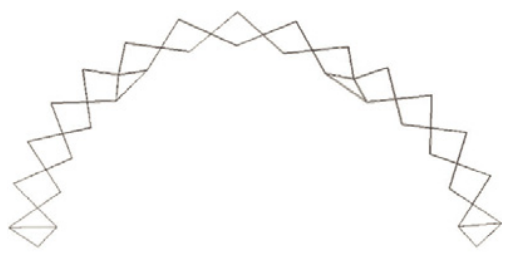

(b) Undeformed and deformed shape.

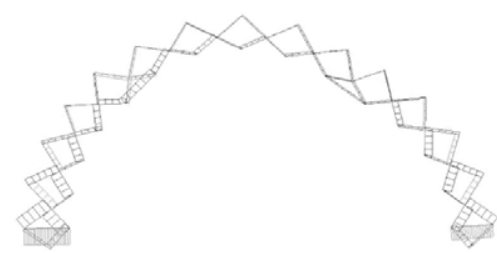

(c) Axial force diagram.

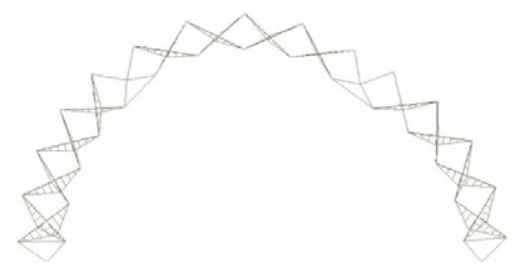

(d) Bending moment diagram.

Fig. 17. Response of high arch with actuators in location 1 subjected to vertical loads.

In the third alternative solution (Fig. 16, locations 3), the structure was connected to the ground via two actuators, while the other two actuators were placed at a suitable position, on the exterior of M-SLEs, so that overall stability is achieved. The vertical deflection was found to be equal to $52 \mathrm{~cm}$, which, however, is unacceptable. The stresses in some cross-sections are unacceptably high as well. The undeformed and deformed shape, as well as the axial force and bending moment diagrams of the structure under vertical loading are shown in Fig. 19.

Satisfactory strength and stiffness were obtained in the fourth solution (Fig. 16, locations 4), which is obtained from solution 3 by adding one more actuator on the top of the arch. This actuator is not needed for deployment and it is only activated for providing increased stiffness of the deployed structure. The total deflection calculated for this case is $8 \mathrm{~cm}$, thus satisfying the serviceability requirement. Stress requirements are also satisfied. The structure is sufficiently stiff, so that geometric nonlinearity is now of limited importance. The undeformed and deformed shape, as well as the axial force and bending moment diagrams of this structure under vertical loading are shown in Fig. 20.

The maximum response quantities of the high arch subjected to vertical loads are summarized in Table 1, for the four alternative locations of actuators. Solution 1 was found to be the best in terms of stiffness and strength, followed closely by solution 4 . Generalizing, actuator locations restricting rotations at the supports, as well as relative rotations between sub-structures, are considered as optimum for resisting gravity loads.

Light weight roofs and structures are particularly sensitive to wind loading, therefore, the structure was also subjected to horizontal loading and geometrically nonlinear analyses were 


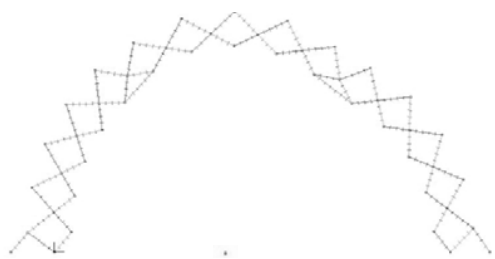

(a) Undeformed shape.

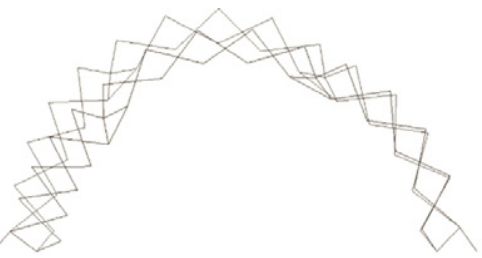

(b) Undeformed and deformed shape.

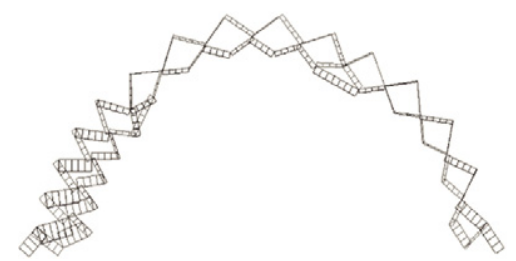

(c) Axial force diagram.

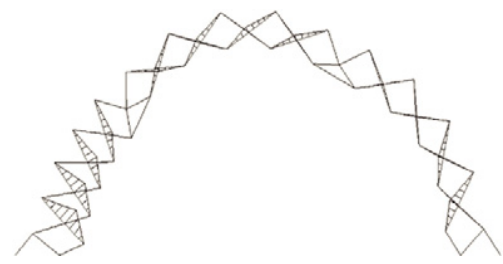

(d) Bending moment diagram.

Fig. 18. Response of high arch with actuators in location 2 subjected to vertical loads.

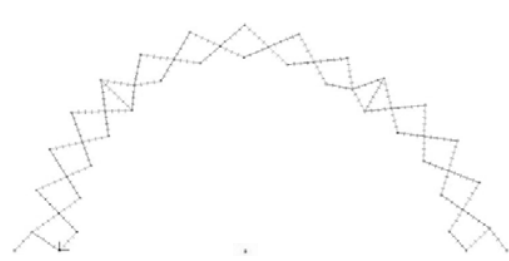

(a) Undeformed shape.

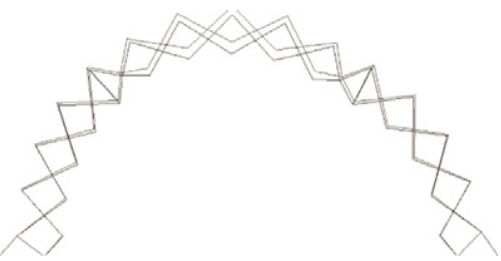

(b) Undeformed and deformed shape.

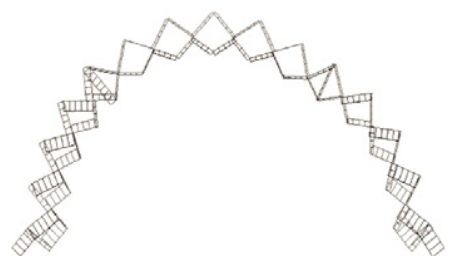

(c) Axial force diagram.

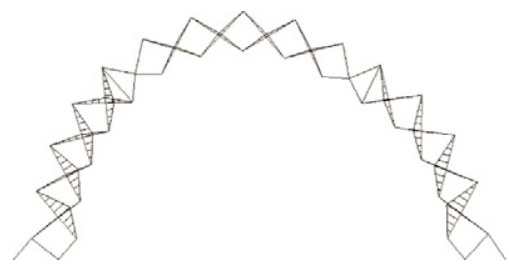

(d) Bending moment diagram.

Fig. 19. Response of high arch with actuators in location 3 subjected to vertical loads.

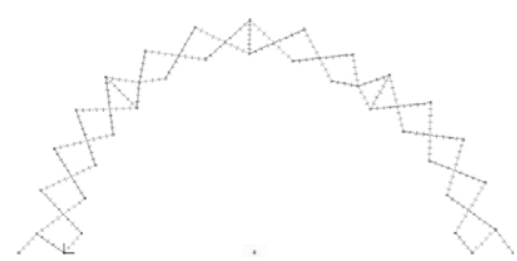

(a) Undeformed shape.

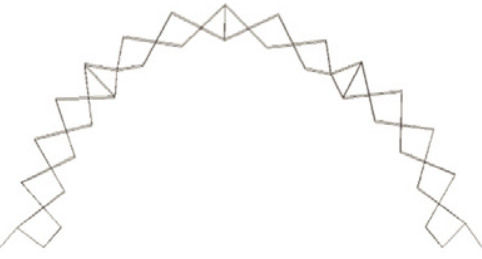

(b) Undeformed and deformed shape.

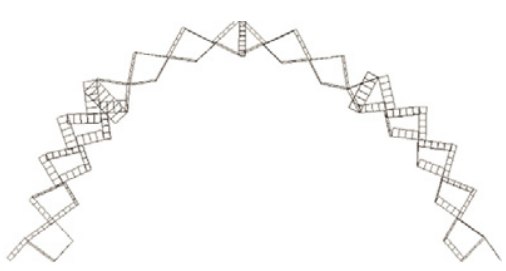

(c) Axial force diagram.

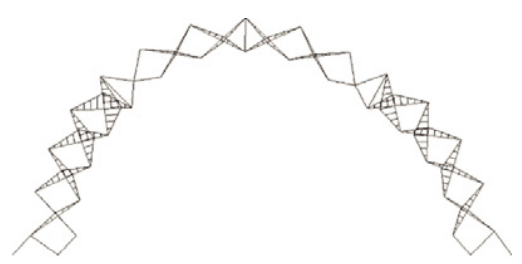

(d) Bending moment diagram.

Fig. 20. Response of high arch with actuators in location 4 subjected to vertical loads.

performed. The wind pressure, corresponding to a wind speed of $26 \mathrm{~m} / \mathrm{s}$, was applied as concentrated loads on the nodes, with a distribution representative of arches subjected to lateral wind, indicated in Fig. 21. The maximum response quantities of the high arch subjected to horizontal loads are summarized in Table 2, for the four alternative locations of actuators. Solution 4 was now 
Table 1

Maximum response quantities of the high arch subjected to vertical loads for the four alternative locations of actuators.

\begin{tabular}{lcc}
\hline Location of actuators & Vertical displacement $(\mathrm{cm})$ & Normal stress $(\mathrm{MPa})$ \\
\hline 1 & 5.2 & 200 \\
2 & 200.0 & 1000 \\
3 & 52.0 & 490 \\
4 & 8.2 & 250 \\
\hline
\end{tabular}

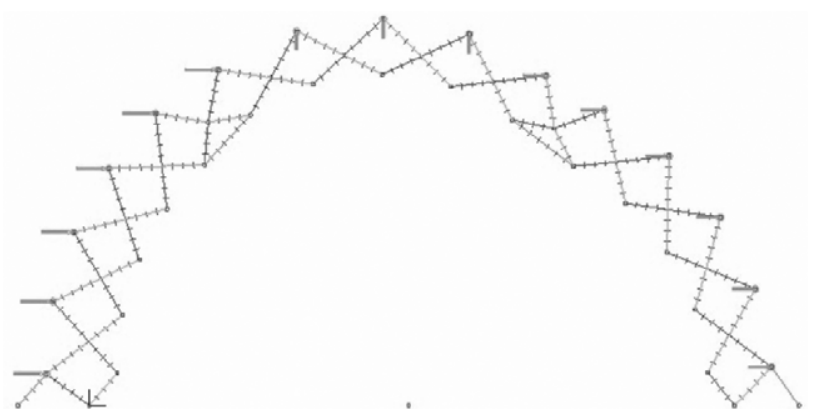

Fig. 21. Distribution of loads representing wind pressure.

Table 2

Maximum response quantities of the high arch subjected to horizontal loads for the four alternative locations of actuators.

\begin{tabular}{lll}
\hline $\begin{array}{l}\text { Location of } \\
\text { actuators }\end{array}$ & $\begin{array}{l}\text { Horizontal } \\
\text { displacement }(\mathrm{cm})\end{array}$ & Normal stress $(\mathrm{MPa})$ \\
\hline 1 & 9.7 & 161 \\
2 & 7.9 & 95 \\
3 & Unstable & - \\
4 & 3.4 & 145 \\
\hline
\end{tabular}

found to be by far the best. Thus, actuator locations restricting rotations at the supports and preventing accordion-type deformation of sub-structures prove to be optimum for resisting horizontal loads.

The same analyses were then carried out for the wavelength arch. The main disadvantages of this shape of structure are the fact that the "arching" action does not exist and that the accumulation of snow on it, in the case that it is used as a roof, will be larger. The results of the analyses, taking snow accumulation into account, are summarized in Tables 3 and 4, for vertical and horizontal loads, respectively. The expected low stiffness and strength are indeed verified. The superiority of the fourth alternative of actuator locations, including a fifth actuator, is demonstrated. Even though there is a slight violation of strength and serviceability criteria, it is proven that with the use of a fifth actuator and with a modest increase of cross-sections, the structure is capable to withstand relatively light loads in this geometry as well. It is also noted that the middle substructure remains at very low levels of stress (Fig. 22), as is also the case for the high arch (Fig. 20), thus savings of material could be possible in that region.

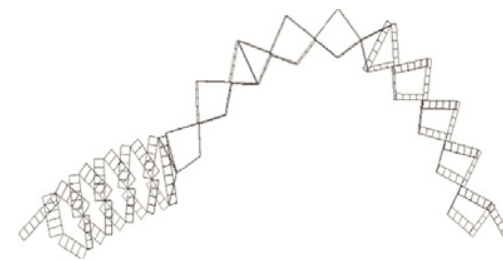

(a) Axial force diagram.
Table 3

Maximum response quantities of the wavelength arch subjected to vertical loads for the four alternative locations of actuators.

\begin{tabular}{lcl}
\hline $\begin{array}{l}\text { Location of } \\
\text { actuators }\end{array}$ & $\begin{array}{l}\text { Horizontal } \\
\text { displacement }(\mathrm{cm})\end{array}$ & Normal stress $(\mathrm{MPa})$ \\
\hline 1 & 75.4 & 800 \\
2 & 390.0 & 900 \\
3 & 20.2 & 410 \\
4 & 15.0 & 340 \\
\hline
\end{tabular}

Table 4

Maximum response quantities of the wavelength arch subjected to horizontal loads for the four alternative locations of actuators.

\begin{tabular}{lcl}
\hline $\begin{array}{l}\text { Location of } \\
\text { actuators }\end{array}$ & $\begin{array}{l}\text { Horizontal } \\
\text { displacement }(\mathrm{cm})\end{array}$ & Normal stress $(\mathrm{MPa})$ \\
\hline 1 & 9.3 & 226 \\
2 & 34.2 & 302 \\
3 & 7.2 & 174 \\
4 & 4.6 & 190 \\
\hline
\end{tabular}

The same analyses were also carried out for the shallow arch. The shallow arch maintains the main disadvantages mentioned in the case of the wavelength arch. The behaviour of the structure under vertical loading approaches more that of a beam and for this reason the static behaviour for such long spans is not satisfactory and it is actually the worst among the three different structural geometries that were analyzed.

\section{Summary and conclusions}

The main principles of a proposed novel concept of planar scissor-hinge structures have been introduced and an analytical framework for their design has been proposed. These structures incorporate a new primary element, the so-called Modified Scissor-Like Element (M-SLE) that enables them to exhibit higher geometric transformation capability than previous designs of scissor-hinge structures. Contrary to previous designs of deployable scissor-hinge structures, in the proposed structures the size of the covered area does not change during transformation. Owing to this property, the proposed structures can be utilized as adaptive permanent roof structures with a wide range of form flexibility. This increased flexibility is achieved by means of actuators, needed for controlling deployment and for stabilizing the structure at a desired configuration, where it can carry loads.

Geometric, kinematic and structural properties of the proposed structure were analyzed in detail. As expected, increased transformability comes at the expense of stiffness and strength. Yet, it has been demonstrated that, by suitable positioning of the actuators, the structure is indeed able to carry low to medium loads in a satisfactory manner. This transformation capability of the proposed planar scissor-hinge structures can in the future be applied to spatial structures based on the same concept, to obtain spatially convertible structures.

Fig. 22. Response of wavelength arch with actuators in location 4 subjected to vertical loads. 


\section{References}

[1] Gantes CJ. Deployable structures: analysis and design. Boston: WIT Press; 2001.

[2] Zuk W, Clark R. Kinetic architecture. New York: Van Nostrand Reinhold; 1970.

[3] Pinero EP. Project for a mobile theatre. Arch Design 1961;12:570.

[4] Escrig F. Expandable space frame structures. In: Nooshin $\mathrm{H}$, editor. Proceeding of the 3rd international conference on space structures. Guildford (UK): Elsevier Applied Science Publishers; 1984. p. 845-50.

[5] Escrig F. Expendable space structures. Space Struct J 1985;2(1):79-91.

[6] Escrig F, Valcarcel JP. Curved expandable space grids. In: Topping BHV, editor Proceeding of the international conference on the design and construction of non-conventional structures. London (England): Civil-Comp Press; 1987. p. $157-68$.

[7] Escrig F, Valcarcel JP. Great size umbrellas with expendable bar structures. In: proceeding of the 1 st international conference on lightweight structures in architecture; 1986 (1). p. 676-81.

[8] Escrig F, Valcarcel JP. Geometry of expendable space structures. Int J Space Struct 1993;8:71-84.

[9] Escrig F, Valcarcel JP. Analysis of expendable space bar structures. In: Heki K editor. Proceeding of IASS symposium on shells, membranes, and space frames, vol. 3. Osaka (Japan): Elsevier Science Publishers; 1986. p. 269-76.

[10] Gantes C, Logcher RD, Connor JJ, Rosenfeld Y. Deployability conditions for curved and flat, polygonal and trapezoidal deployable structures. Int J Space Struct 1993;8:97-106.

[11] Gantes CJ, Logcher RD, Connor JJ, Rosenfeld Y. Geometric design of deployable structures with discrete joint size. Int J Space Struct 1993;8:107-17.

[12] Gantes CJ, Connor JJ, Logcher RD. A systematic design methodology for deployable structures. Int J Space Struct 1994;2(9):67-86.

[13] Gantes CJ, Connor JJ, Logcher RD. Simple friction model for scissor type mobile structures. J Eng Mech 1993;3(119):456-75.
[14] Gantes C, Konitopoulou E. Geometric design of arbitrarily curved bi-stable deployable arches with discrete joint size. Internat J Solids Structures 2004; 41:5517-40.

[15] Langbecker T. Kinematic analysis of deployable scissor structures. Int J Space Struct 1999; $14: 1-15$

[16] Langbecker T, Albermani F. Kinematic and non-linear analysis of foldable barrel vaults. Eng Struct 2001;23:158-71.

[17] Hoberman C. Unfolding architecture: an object that is identically a structure and a mechanism. Arch Design 1993;63:56-9.

[18] You Z, Pellegrino S. Foldable bar structures. Internat J Solids Structures 1997; 15(34):1825-47.

[19] Jensen F, Pellegrino S. Expandable structures formed by hinged plates. In: Parke GAR, Disney P, editors. Proceeding of 5th international conference on space structures, vol. 1. London (UK): Thomas Telford Publishing; 2002. p. 263-72.

[20] Calatrava S. Zur Faltbarkeit von Fachwerken. Ph.D. thesis. Zurich: ETH; 1981.

[21] Tischhauser A, von Moos S. Calatrava: public buildings. Basel (Switzerland): Birkhauser Press; 1998.

[22] Kokawa T. Cable scissors arch-marionettic structure: structural morphology, towards the new millennium. In: Proceeding of international conference of IASS; 1997. p. 107-14.

[23] Akgün Y, Haase W, Sobek W. Proposal for a new scissor-hinge structure to create transformable and adaptive roofs In: Proceeding of international association of spatial structures symposium; 2007.

[24] Alizade R, Bayram C, Gezgin E. Structural synthesis of serial platform manipulators. Mech Mach Theory 2007;42(5):580-99.

[25] Freudenstein F, Alizade R. On the degree of freedom of mechanisms with variable general constraint. In: Proceedings of IFToMM IV. World congress; 1975. p. 51-6.

[26] Söylemez E. Mechanisms. Ankara (Turkey): Middle East Technical University Press; 1999.

[27] Kebiche K, Kazi-Aoual MN, Motro R. Geometrical nonlinear analysis of tensegrity systems. Eng Struct 1999;21(9):864-76. 